\title{
POLYMORPHISM IN SUBDIVIDED POPULATIONS CHARACTERISED BY A MAJOR AND SUBORDINATE DEMES*
}

\author{
SAMUEL KARLIN† and R. B. CAMPBELL $\ddagger$ \\ † Department of Mathematics, Stanford University, Stanford, CA 94305 \\ $\$$ Department of Mathematics, Purdue University, Lafayette, Ind. 47901
}

Received 6.viii.79

\section{Summary}

\begin{abstract}
Subdivided populations where one deme plays a major role and the other demes play subordinate roles are studied. The models are appropriate for socially structured and age structured populations (including plant species with seed pools) as well as spatially subdivided populations. Concise sufficient criteria as well as tractable necessary and sufficient criteria assuring the maintenance of a polymorphism are given. The incorporation of temporal variation is included, and appropriate concepts of environmental heterogeneity are discussed.
\end{abstract}

\section{INTRODUCTION}

In a subdivided population all of the demes may not be of equal importance. Rather there may be one or a few major demes and several subordinate demes. One manifestation of such a dichotomy is the single island model of Wright (1940) which contrasts a stable mainland population with an island population linked by limited migration. The general panmixia model of Levene (1953) generalises the multiple island model of Wright (1951) in which each island receives an equal immigrant fraction from every island by assigning different relative importance to the different islands, but this represents a continuum of deme significance rather than a dichotomy. We focus here on models involving a single major (distinguished) deme which were introduced in Karlin (1976, 1977). Other models reflecting a major/subordinate deme dichotomy include the "star" model of Carmelli and Cavalli-Sforza (1976) and several models in Karlin (1981).

The two models which we consider here entail a linear array of demes connected by unidirectional stepping-stone migration countered by the distinguished deme which either receives immigrants from all or some of the demes (fig. 1) or disperses emigrants to all or some of the demes (fig. 2). Of course the conclusions drawn from these models remain qualitatively valid under small perturbations of the system (Karlin and McGregor, 1972). The physical (geographical) interpretations which we present are hypothetical rather than documented; the non-geographical interpretations are perhaps more important.

The term atoll was used to describe these models (Karlin, 1976) suggestive of a physical array consisting of a major island and several smaller islands extending along a line. Other physical settings include a forest with

* Supported in part by NIH Grant GM10452-16 and NSF Grant MCS 7680624-A01. $44 / 2-$ A 
nearby clusters of trees and shrubs, a stream flowing into (or out of) a lake, and a major city with satellite towns along the main highway. The unidirectional migration could be mediated by wind or water currents, a population density gradient, or the flight patterns of insect vectors. Migration to or from the distinguished deme could be modulated by migratory vectors or different currents countering the unidirectional stepping-stone migration flow.

The distinguished immigrant deme model (fig. 1) is also appropriate for studying age-structured populations. One such example is annual plants which have a seed pool (Templeton and Levin, 1979) (note that their parameterisation is different from ours). The major deme corresponds to the germinating seedlings and the subordinate demes to the age classes in

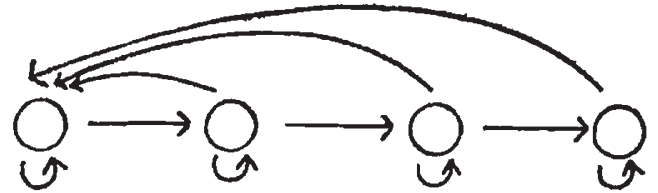

FIG. 1.-Distinguished immigrant deme.

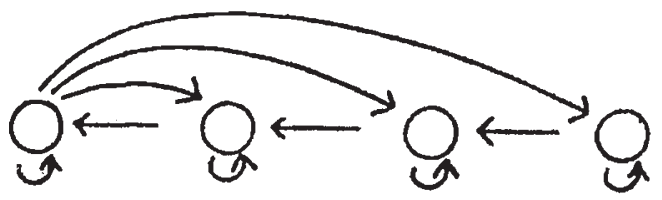

Frg. 2.-Distinguished emigrant deme.

the seed pool (i.e., the seeds are identified with the year they were formed, the age classes are the number of years that they have remained dormant without germinating). A familiar example is the Leslie matrix employed in demographic studies which specifies age-specific birth and death rates. The problem of protection of an allele with different survival probabilities in different age classes can be identified with the problem of growth or extinction of an age-structured population; temporal variation in selection intensities can be identified with temporal variation in birth and death rates.

Most of the results below concentrate on the question of protection of an allele. We present concise sufficient conditions and also sharp criteria assuring maintenance of a polymorphism under constant selection pressures. We then present the formulation and limited results in the presence of deterministic (cyclic) and random temporal variation of the selection intensities. Some comparisons of migration patterns (life histories) and environments with respect to prospects for protection are given. We conclude with the study of some compound migration patterns entailing major and subordinate clusters of demes in their underlying structure.

\section{The Models}

We employ the standard model for local gene frequency changes due to random mating and selection within demes followed by migration among the demes (e.g., Christiansen, 1974; Karlin, 1976). Allele frequency changes within habitats are specified by scalar functions

$$
\hat{x}_{i}=f_{i}\left(x_{i}\right)=\frac{\left[s_{i} x_{i}^{2}+x_{i}\left(1-x_{i}\right)\right]}{\left[s_{i} x_{i}^{2}+2 x_{i}\left(1-x_{i}\right)+t_{i}\left(1-x_{i}\right)^{2}\right]}
$$

(for one locus with two alleles) where the subscript designates the deme or 
habitat. Migration is represented by the backward migration matrix $M$ so that the next generation is given by

$$
x^{\prime}=M \hat{x} .
$$

(The prime (') designates the succeeding generation.) It is the particular form of $M$ which identifies the models which we consider here.

\section{(i) Distinguished immigrant deme}

In this case the backward migration matrix $M$ has the form

$$
M=\left(\begin{array}{cccccc}
a_{1} & a_{2} & a_{3} & a_{4} & \ldots & a_{n} \\
b_{2} & c_{2} & 0 & 0 & \ldots & 0 \\
0 & b_{3} & c_{3} & 0 & \ldots & 0 \\
\vdots & \vdots & . & . & . & . \\
0 & 0 & \ldots & 0 & b_{n} & c_{n}
\end{array}\right) \quad \begin{aligned}
& a_{i} \geqq 0, \sum_{i=1}^{n} a_{i}=1 \\
& i=1,2, \ldots, n
\end{aligned}
$$

The $a_{i}$ specify the fraction of the first deme which is drawn from each deme preceding migration; the $c_{i}$ (and $a_{1}$ ) specify the fraction in each deme which did not migrate and the $b_{i}$ reflect the unidirectional stepping stone component. The specialisation to age-structured populations has $c_{i}=0$, hence $b_{i}=1$ for all $i$ because all members of an age class pass into the next age class with the passage of time.

A frequent question in population genetics relates to the conditions which preclude an allele from extinction. The well-known criterion depends on the spectral radius (magnitude of the largest eigenvalue) of the product matrix $M D$ where $D$ is a non-negative diagonal matrix (all non-diagonal entries are zero) whose entries specify the marginal viability of the allele when rare (e.g., Bulmer, 1972; Christiansen, 1974). The spectral radius of this matrix $M D$ is of course equal to that of $D M$. This latter form is most analogous to the problem of growth or extinction of an age-structured population governed by a specific Leslie matrix. A Leslie matrix has the form

$$
L=\left(\begin{array}{ccccc}
f_{0} & f_{1} & f_{2} & \ldots & f_{n} \\
s_{1} & 0 & 0 & \ldots & 0 \\
0 & s_{2} & 0 & \ldots & 0 \\
0 & 0 & s_{3} & \ldots & 0 \\
\vdots & \vdots & \vdots & & \\
0 & 0 & 0 & \ldots & s_{n}
\end{array}\right)
$$

where $f_{i}$ specifies the birth rate (fertility) associated with the $i$ th age class (which may be zero) and $s_{i}$ is the probability of surviving from the $(i-1)$ st age class into the $i$ th age class. If we let $F=\sum_{i=0}^{n} f_{i}, a_{i}=\frac{f_{i-1}}{F}$, and $b_{i}=1$ 
we see that the growth of a population governed by $L$ is essentially equivalent to protection of an allele in a subdivided population with migration parameters as specified above for (3) and marginal viabilities given by the diagonal matrix

$$
D=\left(\begin{array}{ccccc}
F & 0 & . & . & 0 \\
0 & s_{1} & & \\
\cdot & s_{2} & & \\
\cdot & & \ddots & \\
\cdot & & & \ddots & \\
0 & & & s_{n}
\end{array}\right)
$$

\section{(ii) Distinguished emigrant deme}

The backward migration matrix in this case is essentially a transpose of (3), but of course is constrained to be stochastic, i.e. non-negative entries with row sums equal to one. Specifically, it has the form

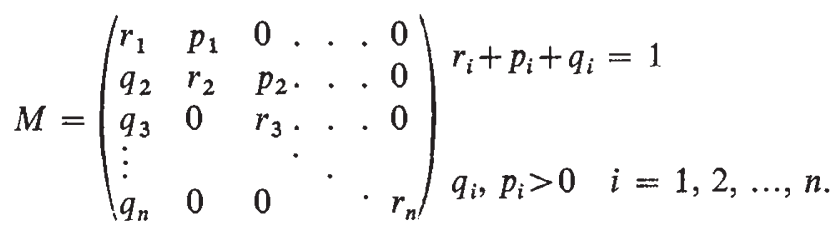

The $q_{i}$ specify the fraction of the $i$ th deme which emigrated from the distinguished (first) deme; $r_{i}$ specifies the sessile fraction; and $p_{i}$ reflects the unidirectional stepping-stone component.

\section{Conditions FOR PROTECTION WITH GONSTANT SELECTION COEFFICIENTS}

The criterion for protection of an allele $A$ (Bulmer, 1972; Christiansen, 1974; Karlin, 1976) is that $\rho(M D)>1$ where $\rho$ designates the spectral radius, $M$ is the backward migration matrix, and $D$ is a diagonal matrix with zeroes off the diagonal and $d_{i i}$ are the marginal viabilities of the $A$ allele on the diagonal (i.e., $d_{i i}$ is the ratio of the viability of the $A a$ heterozygote to the viability of the $a a$ homozygote). A sufficient condition that this spectral radius be greater than one is given in Karlin (1976),

$$
\prod_{i=1}^{n}\left(d_{i i}\right)^{\xi_{t}}>1
$$

where $\underline{\xi}$ is the principal left eigenvector of $M$ normalised such that $\sum_{i=1}^{n} \xi_{i}=1$. The principal left eigenvector of $M$ also appears in other problems of population genetics (e.g., in determining the protection of a recessive allele (Karlin, 1977)). It is therefore of interest to provide the left eigenvector for cases of distinguished deme migration where it has a concise form.

Case (i): For a migration matrix of the form (3) with

$$
0=c_{2}=c_{3}=\ldots=c_{n}
$$


(which is appropriate for an age-structured population) the normalised principal left eigenvector $\underline{\xi}$ is given by

$$
\xi_{i}=\frac{1-\sum_{j=1}^{i-1} a_{j}}{A}
$$

where $A=n-\sum_{j=1}^{n}(n-j) a_{j}$.

Case (ii): For a migration matrix of the form (6) with $r_{2}=r_{3}=\ldots=$ $r_{n}=0$ the principal left eigenvector $\xi$ is given by

$$
\xi_{i}=\frac{R_{i-1}}{\sum_{k=1}^{n} R_{k-1}} \quad i=1,2, \ldots, n .
$$

where $R_{0}=1, R_{i}=\prod_{j=1}^{i} p_{j}$.

(a) Exact criteria. The exact criteria governing protection are available for the immigration and emigration distinguished deme models. Note that $M$ is stochastic but the product matrix $M D$ will in general not be stochastic.

Case $(1 a)$ : Let $M D$ be of the form (cf. (3))

$$
M D=\left(\begin{array}{lllll}
\alpha_{1} & \alpha_{2} & \alpha_{3} & \ldots & \alpha_{n} \\
\beta_{2} & \gamma_{2} & 0 & \ldots & 0 \\
0 & \beta_{3} & \gamma_{3} & \ldots & 0 \\
\vdots & \vdots & & & \vdots \\
& & & \beta_{n} & \gamma_{n}
\end{array}\right)
$$

Then $\rho(M D)>1$ if and only if either

or

$$
\gamma_{i} \geqq 1 \quad \text { for some } i \geqq 2
$$

$$
\gamma_{i}<1 \text { for all } i \text { and } \alpha_{1}+\sum_{k=2}^{n} \alpha_{k} \frac{B_{k}}{R_{k}}>1
$$

where $B_{k}=\prod_{i=2}^{k} \beta_{i}, R_{k}=\prod_{i=2}^{k}\left(1-\gamma_{i}\right)$.

Case (2a): Let $M D$ be of the form (cf. (6))

$$
M D=\left(\begin{array}{llllll}
\gamma_{1} & \beta_{1} & 0 & 0 & \ldots & 0 \\
\alpha_{2} & \gamma_{2} & \beta_{2} & 0 & & \cdot \\
\alpha_{3} & 0 & \gamma_{3} & \beta_{3} & & \cdot \\
& & & \cdot & . & \cdot \\
\alpha_{n-1} & 0 & & & \gamma_{n-1} & \beta_{n-1} \\
\alpha_{n} & 0 & & & 0 & \gamma_{n}
\end{array}\right) .
$$


Then $\rho(M D)>1$ if and only if either

or

$$
\gamma_{i} \geqq 1 \text { for some } i
$$

$$
\gamma_{i}<1 \text { for all } i \text { and } \gamma_{1}+\sum_{k=2}^{n} \alpha_{k} \frac{B_{k}}{R_{k}}>1
$$

where $B_{k}=\prod_{i=1}^{k-1} \beta_{i}, R_{k}=\prod_{i=2}^{k}\left(1-\gamma_{i}\right)$.

Example 1. Let $r_{i}=1-\alpha, p_{i}=\alpha=q_{n}$ in (6). This manifests a form of unidirectional stepping-stone migration (see fig. 3). It may be appropriate for organisms living along ocean currents where a general sedentary tendency is supplemented only by the possibility of movement with the ocean current. Of course, climate and other environmental factors will change

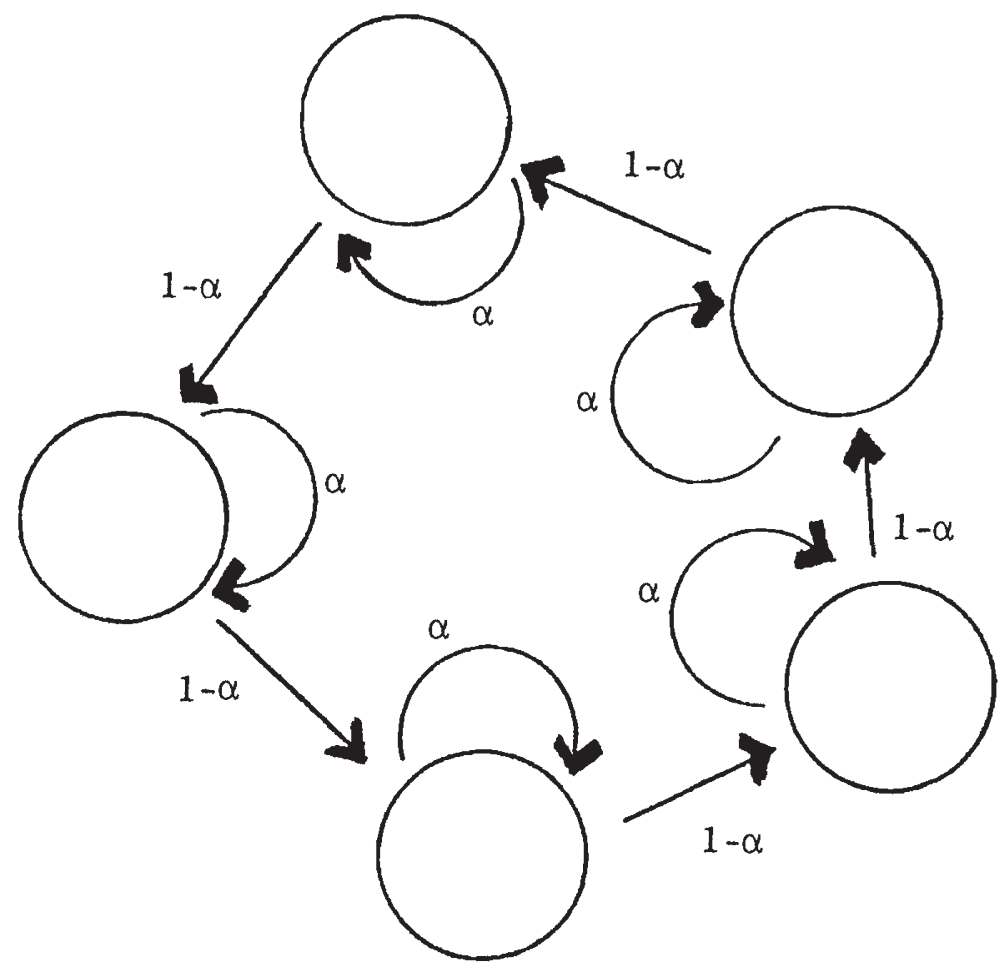

FIG. 3.--The unidirectional stepping stone migration mode of Example 1.

along the course of an ocean current providing variation in selection parameters. Set $D=\operatorname{diag}\left[d_{1}, d_{2}, \ldots, d_{n}\right]$ (i.e., the off diagonal entries are equal to zero and the entries on the diagonal arc as specified), then $\rho(M D)>1$ if and only if either $(1-\alpha) d_{i} \geqq 1$ for some $i$ or the largest positive solution $\lambda_{0}$ of $\prod_{i=1}^{n}\left(\lambda-(1-\alpha) d_{i}\right)=\alpha^{n} \prod_{i=1}^{n} d_{i}$ (which is greater than $\left.\max _{1 \leqq i \leqq n}\left((1-\alpha) d_{i}\right)\right)$ 
exceeds 1 . We shall return to this example later to discuss homing propensities and environmental heterogeneity.

\section{CONDITIONS FOR PROTECTION WITH TEMPORAL SELECTION VARIATION}

\section{(i) Deterministic (cyclic) variation}

Temporal cyclic variation for a system of $n$ demes through $p$ seasons can be represented as selection migration interaction for a system of $n p$ demes without temporal variation. In particular, the backward migration matrix assumes the form

$$
\hat{M}=\left(\begin{array}{lllll}
0 & 0 & \ldots & 0 & M \\
M & 0 & \ldots & 0 & 0 \\
0 & M & \cdot & \cdot \\
\vdots & \ddots & \cdot & \cdot \\
0 & . & . & M & 0
\end{array}\right)
$$

where $M$ is the backward migration matrix per season for the system of $n$ physical demes (actually we may let $M$ vary with the seasons). The selection transformations are analogously transformed into an $n p$ vector

$$
\left\langle f_{1}^{(1)}\left(x_{1}^{(1)}\right), \ldots, f_{n}^{(1)}\left(x_{n}^{(1)}\right), f_{1}^{(2)}\left(x_{1}^{(2)}\right), \ldots, f_{n}^{(p)}\left(x_{n}^{(n)}\right)\right\rangle
$$

where the supercsripts designate the season. This provides an $n p \times n p$ diagonal matrix $\hat{D}$ of marginal viabilities so that protection of an allele can be decided based on $\rho(\hat{M} \hat{D})$. Unfortunately, there is no way to reduce the dimensions of this problem in general.

If the actual migration matrix is constant in time, then the principal left eigenvector of $\widehat{M}$ can be immediately constructed from the principal left eigenvector of $M$ (i.e., $\hat{\xi}=\frac{1}{n}(\underline{\xi}, \underline{\xi}, \ldots, \underline{\xi})$, the $n$-fold catenation of $\underline{\xi}$ ). This provides that in circumstances where the principal left eigenvector of $M$ is available (e.g., (8) and (9)), sufficient conditions for protection (7) under cyclic temporal variation are readily accessible.

\section{(ii) Random temporal variation}

The question of protection is somewhat less tractable under random than deterministic variation. Most studies of random variation in spatially varying environments have assumed either total panmixia as accompanies the Levene migration mode or symmetric migration rates as accompany the Deakin migration mode (e.g., variation in selection in Gillespie (1973, 1975) and in migration in Christiansen (1974)). We specialise to the seed pool model in order to illustrate the formal protection criterion under constant selection intensities contrasted to cyclic variation with particular reference to different modes of selection in populations. We then present a variant model appropriate to populations with seed pools which affords a concise protection criterion under random variation in selection intensities.

The simplest problem involving seed pools entails no selection during the dormant stages as postulated by Templeton and Levin (1979) and constant 
selection intensities with respect to time. In this case protection depends on the spectral radius of a matrix of the form

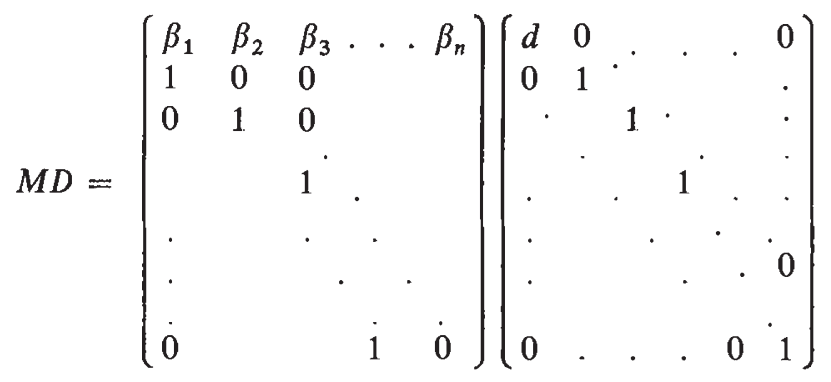

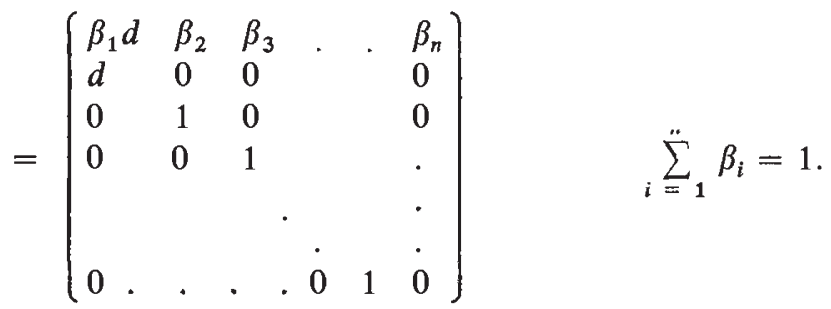

The parameter $d$ reflects the selection on the germinated seeds (first age class) while the dormant age classes experience no selection. The culling of two seed age classes to form the germinating cohort of the next generation and the simultaneous reindexing of the age classes with the passage of time is specified by $M$. It is apparent that protection hinges on whether $d$ is greater than or less than one, but the rate of convergence is a more subtle matter which we shall return to below.

A more realistic formulation allows temporal variation in the selection intensities. This may occur in a deterministic cyclic manner perhaps induced by climatic cycles or induced by man (e.g., crop rotation). The canonical example for cyclical temporal variation is seasonal variation for multivoltine insects, but this example may not be appropriate for seed pools. In these cases the criterion for protection is

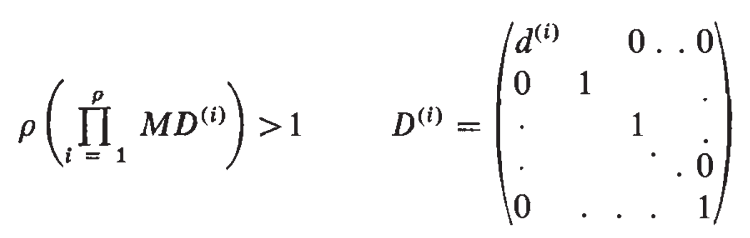

for a $\rho$ generation selection cycle.

Random variation in selection intensities may be more appropriate than deterministic variation because many facets of environmental variation are unpredictable. Under these circumstances the criterion for protection is

$$
\lim _{n \rightarrow \infty}\left(\rho\left(\prod_{i=1}^{n} M D^{(i)^{1 / n}}\right)\right)>1 .
$$

The positing of (18) as the criterion for protection tacitly assumes that the limit exists. For cyclical variation in selection intensities as in (17) it is quite easy to show that the limit exists and is equal to the $n$th root of 
the left-hand side of (17), thereby providing the same criterion for protection. However, it is quite possible to construct temporally varying selection regimes (neither cyclic nor random) such that the limit does not exist. But quite generally for random temporal variation (e.g., selection intensities following a stationary distribution suffices) the limit in (18) does exist as a deterministic limit thereby affording a meaningful protection criterion.

A more general formulation of the seed pool problem which may also be appropriate for other interpretations of distinguished deme migration allows selection during the dormant stages. This case was not treated by Templeton and Levin and their analysis does not extend to encompass it.

The simplest case has temporally constant selection intensities manifesting only two selection regimes: one for the seedlings and one acting on the dormant seeds. In this case the criterion for protection is (17) where $D$ has the form

$$
D=\left(\begin{array}{lllll}
d_{1} & 0 & . & . & 0 \\
0 & d_{2} & . & & . \\
\cdot & . & d_{2} & . & . \\
\cdot & & \cdot & \cdot & . \\
. & & & \cdot & .0 \\
0 & . & . & 0 & d_{2}
\end{array}\right) .
$$

A more general formulation allows $D$ to be a general diagonal matrix

$$
D=\left(\begin{array}{llllll}
d_{1} & 0 & & & & 0 \\
0 & d_{2} & & & \\
\cdot & \cdot & d_{3} & & \cdot \\
\cdot & & \cdot & \cdot & & \cdot \\
\cdot & & & \cdot & \cdot & 0 \\
0 & \cdot & . & & 0 & d_{n}
\end{array}\right) .
$$

This stipulates that the selection intensities depend on the number of years that the seeds have remained dormant which may be difficult to interpret in the context of seed pools, but is quite reasonable for other distinguished deme interpretations.

Interpretation of systematic or random temporal variation follows analogously to (17) or (18) where the $D^{(i)}$ have the form (19) or (20).

Remark. The above cases have assumed that the backward migration matrix $M$ is constant in time. This may not be a reasonable assumption, especially if the total seed production fluctuates greatly from year to year. The incorporation of temporal variation in the backward migration matrix is achieved by superscripting the migration matrices as well as the selection regimes in (17) and (18) to allow for temporal migration variation.

For the case of temporally constant selection intensities (16) it is of interest to ask how rapidly the fixation state is approached (if stable) which corresponds to calculating a Malthusian parameter for the rate of decrease of the rare allele. To address the problem it is convenient to consider the matrix product in (16) in the order DM. The Malthusian parameter is the log of the spectral radius of the matrix $D M$ which is the log of the largest positive root of

$$
-\lambda^{n}+d \sum_{i=1}^{n} \beta_{i} \lambda^{n-i}=0
$$


With the definition

$$
\bar{G}=\sum_{i=1}^{n} i \beta_{i}
$$

Templeton and Levin noted that the Malthusian parameter is approximately

$$
[d-1] / \bar{G}
$$

for weak selection, i.e. for $d$ close to 1 .

For convenience we study the spectral radius of $D M$ rather than its log. From (21) an approximate spectral radius is given by

$$
\left[1+\frac{(n-1)(1-d)}{\bar{G} d}\right] /\left[1+\frac{n(1-d)}{\bar{G} d}\right] \approx d^{1 / G}
$$

for weak selection. Thus we see that $\bar{G}$ which gives the mean length of dormancy serves to alter the convergence rate to that corresponding to temporal variation with selection acting once every $\bar{G}$ generations in accordance with (23).

How does the left-hand side of (24) bound the spectral radius? For weak selection our approximating root is an upper bound on the spectral radius of $D M$, in particular $d>\sqrt{\frac{n-2}{n}}$ is a sufficient condition for this to be true (if $d<1$, which assumes stability).

Example. Consider the migration pattern (3) with $c_{2}=c_{3}=\ldots=$ $c_{n}=0$ and selection acting only on the first (immigrant) deme. This is appropriate for the study of plants with seed pools where selection acts only on the germinated plants and not on the dormant seeds. Under a slight alteration of our hypotheses we have a concise result on protection.

Instead of a finite-lived seed pool as is characterised by a backward migration matrix $M$, we postulate an infinite-lived seed pool with the germination probability of age classes decaying exponentially with age. In particular, we assume that one half of the germinating seeds came from the current plants and $(1 / 2)^{i}$ of the germinating seeds came from the $i$ th age class in the seed pool (the current plants are the first age class). The basic transformation equation is

$$
x_{1}^{\prime}=\frac{1}{2} x_{1} d_{1}+\sum_{j=2}^{\infty}\left(\frac{1}{2}\right)^{j} x_{j},
$$

where the prime $\left({ }^{\prime}\right)$ designates the next generation. Under the circumstance that the marginal selection coefficient $d_{1}$ varies randomly in time the sharp criterion for protection is

$$
E\left[\ln \left(1+d_{1}\right)-\ln (2)\right]>0
$$

where E[.] designates the expected value (we show this in the appendix).

It is elementary to show that

$$
\frac{1}{2} E\left[\ln \left(d_{1}\right)\right] \leqq E\left[\ln \left(1+d_{1}\right)-\ln (2)\right] \leqq E\left[\frac{1+d_{1}}{2}\right]-1 .
$$

Therefore, the sharp criterion for protection in an infinite-lived seed pool lies between the criteria for pure spatial variation (Levene, 1953) and pure temporal variation (Deakin, 1966) buttressing the notion that seed pools manifest a cross between spatial and temporal variation. 
We also note that this formulation of seed pools can be posed as a twodeme model recognising the active plant environment versus the dormant seed phase.

We remark that the assumption that there is no selection in the seed pool is not intrinsic to the above calculations, but rather the assumption that the same selection forces are acting on all age classes in the seed pool during a given time span. The above calculations are appropriate employing the ratio of the marginal viabilities in the active to dormant demes if the result is adjusted by the expected value of the log of the marginal viabilities in the dormant age classes.

The first row of the backward migration matrix (3) with the specialisation $c_{2}=c_{3}=\ldots=c_{n}=0$ corresponding to plants with seed pools is based on the distribution of the number of years that seeds remain in the seed pool before germinating. The number of years until germination is of course a random variable, but may depend on the genotype of the seed; perhaps at loci independent of the locus where selection is being studied. Under this circumstance the time until germination of seeds in the seed pool is given by the compound distribution resulting from the probability of the background genotypes and the duration of dormancy conditioned on the background genotype.

(a) Environmental heterogeneity. For some cases of these distinguished deme migration patterns we can consider the problem of a partial ordering of environments with respect to heterogeneity in the sense that if environment $\varepsilon^{\prime}$ is an appropriate average of environment $\varepsilon$, then it entails less heterogeneity and the prospects for protection of alleles are lessened (cf. Karlin, 1980). We highlight two such cases.

Case $(1)$ : We turn our attention to the unidirectional circular steppingstone pattern of example 1 which is a specialisation of the distinguished emigrant deme model. (Reversing the direction of the migration makes it a special case of the distinguished immigrant deme model and all the attendant arguments follow mutatis mutandis.) Since $M$ is doubly stochastic, we shall consider more heterogeneous environments defined by

$$
\underline{s}^{\prime}=T \underline{s}, \underline{t}^{\prime}=T \underline{t}
$$

where the matrix $\mathcal{T}$ is doubly stochastic in accordance with Karlin (1976) and $s_{i}$ and $t_{i}$ give the relative viabilities of the two homozygous types in the respective demes. We show in the appendix that this is indeed an appropriate concept of environmental averaging in the sense that prospects for a protected polymorphism are less under the averaged selection regime.

Case (2): We specialise (6) to the case with $r_{i}=0$ for all $i$ (i.e., the entries on the diagonal of $M$ are equal to zero). For an environmental averaging process we again consider $\underline{s}^{\prime}=T \underline{s}, \underline{t}^{\prime}=T \underline{t}$ where $T$ is doubly stochastic. In this circumstance the notion of environmental average is not the one posited by Karlin (1976) since $M$ is not doubly stochastic.

However, under the further assumption that $\underline{s}$ and $\underline{t}$ are monotone increasing vectors (the marginal viabilities specified by $d$ form a decreasing vector), the prospects for a protected polymorphism decrease with a more uniform (less heterogeneous) environment. In contrast, if the components of $\underline{s}$ and $\underline{t}$ are decreasing ( $d$ is increasing) the prospects for protection increase with a more uniform environment. 
(b) Generalisations (clustering). We have discussed above the method of converting $\rho$ seasonal temporal variation superimposed on an $n$ age-class seed pool into an $n \rho$ deme migration problem. In particular, we noted that the left eigenvector for $\hat{M}$ (14) was as accessible as the left eigenvector for $M$ and hence sufficient conditions assuring protection are available. We consider some other extensions.

Uniform migration among age structured populations. If all age classes of a population subdivided into $k$ demes experience total panmixia (weighted by the constant relative sizes of the demes), then the migration/aging process can be represented as a $k n \times k n$ matrix which is the Kronecker (tensor) product of the underlying Levene and distinguished deme migration structures

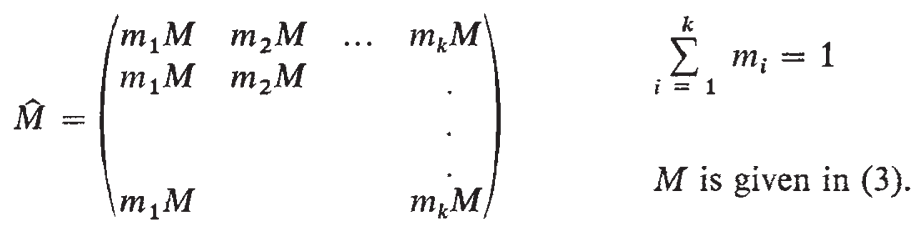

This model is appropriate for the study of seed pools in subdivided populations since although there is no migration among the dormant stages, the lack of selection during dormancy and rank one migration structure make the migrations during dormancy superfluous (a single migration entails total mixing).

If the overlying spatial migration structure is Deakin or in fact any structure for which the left eigenvector is known, the left eigenvector for the composite matrix is of course immediately accessible, but interpretations in terms of the seed pool model are more tenuous.

\section{Discussion}

The recognition that demes in a subdivided population will assume different roles relative to the genetic structure of the population as a whole has led to the formulation of several distinguished deme population structures emphasising various ways in which these different roles may be manifested. Besides providing insight into the behaviour of spatial genetic systems from the behaviour of extreme cases, they are also descriptive of circumstances governing age structured populations. In particular, the life histories of many annual plants whose seeds experience a variable dormancy period could be described by selection-migration structures of this nature. They are also related to the Leslie matrices employed in demographic studies, in particular, the question of growth versus extinction of an age-structured population can be reduced to the question of protection of an allele with a distinguished deme migration structure. We emphasise several facets pertaining to these distinguished deme migration structures.

\section{(i) Protection}

Conditions assuring the maintenance of a polymorphism are of interest because of the pervasiveness of polymorphism in nature and have been 
studied by many authors (see the reviews by Felsenstein, 1976 and Hedrick, Ginevan and Ewing, 1976). In this paper we provide concise sufficient criteria for protection for several of the models, see formulas (7-9), (24), (27). The sharp criteria governing protection are also available for all of the migration structures we consider (e.g. formulas (11), (12), (13a, b), (17), (18), 26)).

\section{(ii) Temporal variation}

Variation in selection intensities will obviously increase the complexity of the problem. In the case of deterministic cyclic variation a concise formulation of the problem is available and for the specific model corresponding to age-structured populations essentially the same sufficient criterion for protection is available as in the case of temporally constant coefficients. Random variation in selection intensities leaves the problem less suited to analysis, although still tractable but an analogous formulation of the seed pool problem which has equal biological justification allows a very concise sharp criterion for protection (e.g., see (17), (18), (24), (26)).

\section{(iii) Clustering}

The superposition of temporal variation on distinguished deme migration resulted in a "migration" matrix which is the Kronecker (tensor) product of the migration matrices corresponding to distinguished deme migration and temporal selection variation (see (14)). Superimposing spatial variation and migration on age-structured populations produced migration structures represented by the Kronecker product of the geographic and agestructure migration matrices (see (29)). The tensor product structure simplifies consideration of many questions, especially sufficient conditions for protection. There are also many cases where pure migration can be broken down to a hierarchy of levels which can be represented as the Kronecker product of lower dimensional migration matrices rendering analyses more tractable.

\section{(iv) Environmental heterogeneity}

The question of degree of environmental heterogeneity and the prospects for protection was considered for some of the distinguished deme models. In the case of unidirectional stepping-stone migration the notion of a more averaged environment given in Karlin (1976) indeed provides less prospects for maintenance of a polymorphism. However, for the distinguished deme model precluding the possibility of individuals remaining in their original habitat after migration, there are diverse possibilities depending on the nature of the selection regime. If the selection regime represents a monotone cline in selection intensities, then an averaged environment provides greater or less prospects for protection depending on the direction of the cline.

\section{REFERENCES}

Bulmer, m. G. 1972. Multiple niche polymorphism. Am. Nat., 106, 254-257. GARMELLI, D., AND CAVALLI-SFORZA, L. L. 1976. Some models of population structure and evolution. Theor. Pop. Biol., 9, 329-359. 
CHRISTIANSEN, F. B. 1974. Sufficient conditions for protected polymorphism in a subdivided population. Am. Nat., 108, 157-164.

DEAKIN, M. A. B. 1966. Sufficient conditions for genetic polymorphism. Am. Nat., 100, $690-692$.

FELSENSTEIN, J. 1976. The theoretical population genetics of variable selection and migration. Ann. Rev. Genet., 10, 253-280.

gillespie, J. H. 1973. Polymorphism in random environments. Theor. Pop. Biol., 4, 193-195.

GILLESPIE, J. H. 1975. The role of migration in the genetic structure of populations in temporally and spatially varying environments. I. Conditions for polymorphism. Am. Nat., 109, 127-135.

HEDRICK, P. W., GINEVAN, M. E., AND EWING, E. P. 1976. Genetic polymorphism in heterogeneous environments. Ann. Rev. Ecol. Syst., 7, 1-32.

KARLIN, s. 1976. Population subdivision and selection migration interaction. In Population Genetics and Ecology, eds. S. Karlin and E. Nevo, pp. 617-657. Academic Press, New York.

KARLIN, S. 1977. Protection of recessive and dominant traits in a subdivided population with general migration structure. Am. Nat., 111, 1145-1162.

KARLIN, s. 1981. Theoretical Population Genetics. Academic Press, New York (in preparation). KARLIN, s., AND MCGREGOR, J. L. 1972. Application of method of small parameters to multiniche population genetic models. Theor. Pop. Biol., 3, 186-209.

Karlin, s., And taylor, H. M. 1975. A First Course in Stochastic Processes. New York: Academic Press.

LEVENE, H. 1953. Genetic equilibrium when more than one ecological niche is available. Am. Nat., 87, 331-333.

TEMPLeton, A. R., AND LeVIN, D. A. 1979. Evolutionary consequences of seed pools. Am. Nat., 114, 232-249.

WRIGHT, s. 1940. Breeding structure of populations in relation to speciation. Am. Nat., $74,232-248$.

WRIGHT, s. 1951. The genetical structure of populations. Ann. Eugen., 15, 323-354. 


\section{Appendix A}

(Proof of necessity and sufficiency of (11) and (12)).

Theorem. Let $G$ be an irreducible nonnegative matrix of the form (10). Then $\rho(G)>1$ if and only if either (let $\left.\alpha_{1}=\gamma_{1}\right)$

$$
\gamma_{i} \geqq 1 \text { for some } i
$$

or

$$
\gamma_{i}<1 \text { for all } i \text { and } \gamma_{1}+\sum_{k=2}^{n} \alpha_{k} \frac{B_{k}}{\prod_{i=1}^{k}\left(1-\gamma_{i}\right)}>1 \text { holds. }
$$

Proof. (Sufficiency). Where condition (A.1) applies, sufficiency follows from an elementary characterisation of the spectral radius for a non-negative matrix (see, e.g., the appendix to Karlin and Taylor, 1975). so that

Let $\underline{x}=\left(x_{1}, x_{2}, \ldots, x_{n}\right)$ be a right positive eigenvector for $\lambda_{0}=\rho(G)$

or

$$
x_{k+1}=x_{k} \frac{\beta_{k+1}}{\lambda_{0}-\gamma_{k+1}}, \quad k=1,2, \ldots, n-1
$$

$$
x_{k+1}=x_{1} \frac{B_{k+1}}{R_{k+1}}, k=1, \ldots, n,
$$

where $B_{1}=1, R_{1}=1$ for convenience and

$$
B_{k}=\prod_{i=2}^{k} \beta_{i}, \quad R_{k}=\prod_{i=2}^{k}\left(\lambda_{0}-\gamma_{i}\right), \quad k \geqq 2 .
$$

Expressing the eigenvector equation for the component $k=1$ leads to the implicit formula

provided $x_{1}>0$. Observe that

$$
\lambda_{0}=\sum_{k=1}^{n} \alpha_{k} \frac{\prod_{i=2}^{k} \beta_{i}}{\prod_{i=2}^{k}\left(\lambda_{0}-\gamma_{i}\right)}
$$

$$
R_{k}(\lambda)=\frac{1}{\prod_{i=2}^{k}\left(\lambda-\gamma_{i}\right)}
$$

decreases convexly from infinity to zero as $\lambda$ increases from $\max _{2 \leqq i \leqq n} \gamma_{i}$ to $\infty$.

Hence, in the circumstances of $\gamma_{i}<1$ for all $i, \lambda_{0}$ is the unique solution of (A.6) exceeding $\max _{1 \leqq i} \gamma_{i}$ which by (A.2) and (A.6) exceeds 1 . We can calculate $x_{k}, k \geqq 2$ from (A.4) normalising $x_{1}=1$. On account of the foregoing fact, we derive easily the relations of $G \underline{x}=\lambda_{0} \underline{x}$ and accordingly $\lambda_{0}=\rho(G)$.

(Necessity). If $\rho(G)>1$ and (A.1) does not apply then indeed (A.6) holds with $\lambda_{0}=\rho(G)>1$ implying the necessity of (A.2).

The proof for (13) follows mutatis mutandis considering the left principal eigenvector. 
(Proof of necessity and sufficiency of (26)). The transformation (25) corresponds to a census time at germination. The question of protection is more accessible from the transformation

$$
\hat{x}_{1}^{\prime}=d_{1} \sum_{j=1}^{\infty}\left(\frac{1}{2}\right)^{j} \hat{x}_{j}
$$

which corresponds to a census time at seed set (before migration in the spatial interpretation). This equation (A.7) suggests introducing the variable

for which

$$
\bar{x}=\sum_{j=1}^{\infty}\left(\frac{1}{2}\right)^{j} \hat{x}_{j}
$$

$$
\bar{x}^{\prime}=\frac{1}{2}\left[d_{1} \bar{x}+\bar{x}\right] .
$$

This equation provides through iteration

$$
\bar{x}^{(t)}=\left(\prod_{i=1}^{t}\left(\frac{1}{2}\left(d_{1}^{(i)}+1\right)\right)\right) \bar{x}^{(0)}
$$

where the superscript designates the generation. Hence protection ensues if

$$
E\left[\ln \left(1+d_{1}\right)-\ln (2)\right]>0
$$

which is (26) as was to be shown.

(Proof that an averaged environment (28) provides less prospects for protection for the migration pattern of Example 1.) We show that (28) provides a useful concept of environmental averaging for the migration pattern of Example 1. The specialisation $n_{i}=1-d, \rho_{i}=\alpha$ of (13) for this migration pattern provides that $\rho(M D)>1$ if either

$$
(1-\alpha) d_{i} \geqq 1 \text { for some } i
$$

or

$$
(1-\alpha) d_{i}<1 \text { for all } i \text { and } \lambda^{+}>1
$$

where $\lambda^{+}$is the largest positive solution of

$$
\prod_{i=1}^{n}\left(\lambda-(1-\alpha) d_{i}\right)=\alpha^{n} \prod_{i=1}^{n} d_{i}
$$

The averaging criteria (28) provide that the marginal viabilities satisfy

$$
\frac{1}{d_{i}^{\prime}}=\sum_{j=1}^{n} t_{i j} \frac{1}{d_{j}} \text { or }\left(\frac{1}{\underline{d}^{\prime}}\right)=T\left(\frac{1}{\underline{d}}\right) .
$$

Thus we shall prove if $\rho(M D) \leqq 1$ then $\rho\left(M D^{\prime}\right) \leqq 1$ where $D=\operatorname{diag}[d]$ and $D^{\prime}=\operatorname{diag}\left[\underline{\underline{d}}^{\prime}\right]$ as specified in (28) where $T$ is doubly stochastic.

For the proof we assume hereafter

$$
\rho(M D) \leqq 1
$$

It is known (by virtue of the concavity of the log function) that

$$
\prod_{i=1}^{n} a_{i}^{\prime} \geqq \prod_{i=1}^{n} a_{i} \quad \text { when } \quad \underline{a}^{\prime}=T_{a}
$$


with $T$ doubly stochastic, and strict inequality prevails unless the elements of $\left\{a_{i}\right\}$ and $\left\{a^{\prime}\right\}$ are identically a fixed constant or $T$ is a permutation matrix. Invoking the fact (A.I7) we deduce under the relationships (28) that

$$
\prod_{i=1}^{n} d_{1}^{\prime}=\prod_{i=1}^{n} \frac{1}{\left(T \frac{1}{d}\right)_{i}} \leqq \frac{1}{\prod_{i=1}^{n} \frac{1}{d_{i}}}=\prod_{i=1}^{n} d_{i}
$$

carrying strict inequality provided $T$ is not a permutation matrix and $\underline{\underline{d}}$ is not a constant regime.

Consider the situation where

$$
(1-\alpha) d_{i} \leqq 1 \text { for all } i
$$

as consistent with the assumption $\rho(M D) \leqq 1$ equivalent to the conditions (11). Since

$$
d_{i}^{\prime}=\frac{1}{\left(T \frac{1}{d}\right)_{i}}<(T \underline{\underline{d}})_{i}, i=1,2, \ldots, n
$$

it follows that

$$
(1-\alpha) d_{i}^{\prime}<1 \text { for all } i .
$$

Observe that for $\lambda>(1-\alpha) \max _{1 \leqq i \leqq n} d_{i}$ and $\underline{e}=(1, \ldots, 1)$ then

$\prod_{i=1}^{n}\left[\lambda-(1-\alpha)(T \underline{\underline{d}})_{i}\right]=\prod_{i=1}^{n}(T(\lambda \underline{\underline{e}}-(1-\alpha) \underline{\underline{d}}))_{i} \geqq \prod_{i=1}^{n}\left(\lambda-(1-\alpha) d_{i}\right)$

by (A.17). We observed in (A.20) that $d_{i}^{\prime}<(T d)_{i}$ and the relations (A.17) and (A.22) together entail

$\prod_{i=1}^{n}\left(\lambda-(1-\alpha) d_{i}^{\prime}\right)>\prod_{i=1}^{n}\left(\lambda-(1-\alpha)\left(T \underset{\underline{d}}{d_{i}}\right) \geqq \prod_{i=1}^{n}\left(\lambda-(1-\alpha) d_{i}\right)\right.$.

The conjunction of (A.18) and (A.23) show that for $\lambda_{0}=\rho(M D)$,

$\prod_{i=1}^{n}\left(\lambda_{0}-(1-\alpha) d_{i}^{\prime}\right) \geqq \prod_{i=1}^{n}\left(\lambda_{0}-(1-\alpha) d_{i}\right)=\alpha^{n} \prod_{i=1}^{n} d_{i}>\alpha^{n} \prod_{i=1}^{n} d_{i}^{\prime}$.

We infer from (A.24) that the equation

$$
\prod_{i=1}^{n}\left(\lambda-(1-\alpha) d_{i}^{\prime}\right)=\alpha^{n} \prod_{i=1}^{n} d_{i}^{\prime}
$$

is satisfied for $\lambda^{\prime}=\rho\left(M D^{\prime}\right)<\rho(M D)$. The proof is complete.

(Implications of the average (28) with no homing.) We consider the special case of (6) with $r_{i} \equiv 0$. Let

$$
P_{i}=\prod_{k=1}^{i} p_{k}, \quad i \geqq 1, \quad P_{0}=1 \quad \text { and } \quad D_{i}=\prod_{k=1}^{i} d_{k},
$$

then $A$-protection, that is $\rho(M D)>1$, cf. (13b), occurs if and only if

$$
\sum_{i=1}^{n} a_{i}>1 \text { where } a_{i}=q_{i} P_{i-1} D_{i}
$$

$44 / 2-B$ 
Consider an environment induced by the marginal selection parameters $\left\{d_{1}^{\prime}, d_{2}^{\prime}, \ldots, d_{n}^{\prime}\right\}$ defined from the parameters $\left\{d_{1}, d_{2}, \ldots, d_{n}\right\}$ in (28). Note that

$$
\sum_{i=1}^{n} \frac{1}{d_{i}}=\sum_{i=1}^{n} \frac{1}{d_{i}^{\prime}}
$$

because $T$ is doubly stochastic. It is an elementary mathematical result that if $\left\{d_{i} \downarrow\right\}\left(d_{i}\right.$ decreases), then (cf. A.18)

$$
D_{i}^{\prime} \leqq D_{i}, \quad i=1,2, \ldots, n .
$$

These relations applied in the presence of condition (A.27) establish that for the migration pattern (6) with $r_{i} \equiv 0$, the phenomenon of protection of the $A$-allele is enhanced with increasing environmental heterogeneity (in the sense of (28)) provided the selection gradient is monotone increasing. For the pattern $d_{i} \uparrow$ the inequalities of (A.29) are reversed and the prospects of $A$-protection are reduced. 